\title{
Physician and patient treatment decision-making in relapsing-remitting multiple sclerosis in Europe and the USA
}

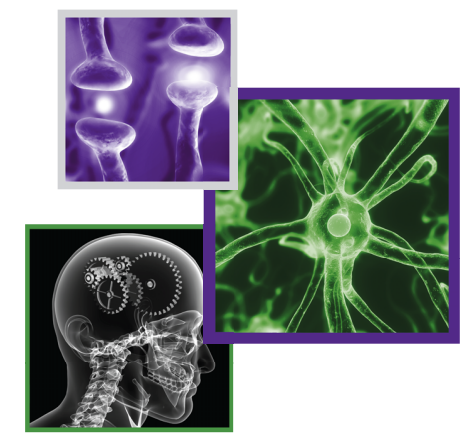

\author{
Hannah Brown*,1, Simone Gabriele ${ }^{1}$ \& Joanna White ${ }^{1}$ \\ ${ }^{1}$ Ipsos Healthcare, London \\ *Author for correspondence: Tel.. + 44 (0) 203059 5056; hannah.brown@ipsos.com
}

\section{Practice points}

- Treatment decisions were largely made jointly between physicians and patients across both the 5 EU (UK/Germany/France/Italy/Spain) and the USA.

- Decisions remain largely efficacy focused, driven by efficacy for relapses.

- Patients are more engaged earlier in the treatment pathway, and this decreases as they progress through treatment lines.

Objective: To assess factors used in treatment decision-making for relapsing-remitting multiple sclerosis in both the 5EU (UK/Germany/France/Italy/Spain) and the USA. Methods: Data were abstracted from the Ipsos Healthcare Global Multiple Sclerosis Therapy Monitor (data abstracted September-December, 20152017) a bi-annual online chart-review study of patients with multiple sclerosis. Results: Treatment choices were primarily due to efficacy for relapses while convenience factors, other than oral administration, were uncommon. Predominantly, the choice of disease modifying therapy at first line was jointly made, with patient involvement decreasing at later lines of treatment. Conclusion: Relapsing-remitting multiple sclerosis patients are highly engaged in their treatment decisions, particularly early in the pathway. The majority were made jointly between the physician and patient, driven by efficacy.

First draft submitted: 22 May 2018; Accepted for publication: 11 September 2018; Published online: 19 November 2018

Keywords: decision-making • efficacy • patient engagement • relapsing-remitting multiple sclerosis • RRMS

The therapeutic landscape for relapsing-remitting multiple sclerosis (RRMS) has evolved rapidly over the past 15 years as an expansion in treatment options has reshaped the clinical approach to therapy [1]. A greater pool of disease modifying therapies (DMTs) brings a rise in expectations for both physicians and patients. Historically, efficacy, the ability of a therapy to reduce relapse rate or disability progression, primarily drove DMT selection. However, the longevity and variable disease course of multiple sclerosis (MS) necessitates a treatment with the additional factors of safety and convenience as the importance of quality of life is identified as a clinical end point [2]. In a preference-sensitive condition such as RRMS, the availability of such choice in therapy allows for treatment decisions to be made jointly by physicians and patients as patient-centered goals and risks shape the therapeutic approach [3].

With little known regarding variances in patient engagement as a patient progresses through treatment, the purpose of the present study was to obtain insight of this regard alongside the treatment drivers behind decisionmaking as a patient progresses through both lines of therapy and disease severity.

\section{Materials \& methods}

\section{Study design}

The Ipsos Healthcare Global Multiple Sclerosis Therapy Monitor is a multiyear, multicenter retrospective chartreview study of RRMS patients, conducted in the 5EU (UK/Germany/France/Italy/Spain) and the USA since 1997. Approximately 370 physicians (270 5EU, 100 US) provided retrospective de-identified data, online, on the next ten consecutive MS patients that they saw for a consultation. 
The collected data elements included patient demographics and clinical status, diagnosis/treatment patterns and disability status. RRMS patients currently treated with DMTs were evaluated.

Patient data from the ten consecutive MS patients were documented by physicians with the assistance of a set of online questions referred to in the study as a patient record form in the following ratio: seven RRMS, one secondary progressive multiple sclerosis (SPMS), one primary progressive multiple sclerosis (PPMS) and one clinically isolated syndrome (CIS) patient, during the study observation period. Inclusion criteria denoted that for each wave, physicians were randomly recruited from a large panel to enable geographic representativeness. Each physician was specified to satisfy the following criteria: see $\geq 15$ MS patients/month; write $>1$ prescription for a DMT per month; are responsible for the long-term treatment decisions of their MS patients and have experience in managing MS for a minimum of 3 years. Approximately $70 \%$ retention of physicians was achieved between waves but patients were newly recorded each wave, in other words, the data are not longitudinal.

Although high physician retention allowed for a robust view of trends over time, there is some variation in participants, and the patient population evaluated provided a cross-sectional review of their practices at the given time window(s).

\section{Statistical analysis}

Descriptive statistical methods were utilized to analyze the data corresponding to the factors used in treatment decision-making for RRMS patients in Q4 2017 for 5EU and the USA.

\section{Results}

In total, 1586 (Q4 2015), 1591 (Q4 2016), 1698 (Q4 2017) and 688 (Q4 2015), 602 (Q4 2016), 663 (Q4 2017) charts of eligible RRMS treated patients were abstracted across 5EU and the USA, respectively. Over the 2015 to 2017 time period, treatment decisions among RRMS patients were predominantly based on 'efficacy for relapses' as the primary treatment driver in both the 5EU and USA (5EU: 30.4\% Q4 2015/29.4\% Q4 2017. USA: 27.6\% Q4 2015/27.3\% Q4 2017). Other major considerations in Q4 2017 included slows progress of disability' (5.4\%/17.8\%), 'efficacy in early MS' (12.3\%/9.2\%), 'best benefit-to-risk profile' (5.4\%/6.0\%) and 'oral administration' (8.5\%/6.0\%) in 5EU and the USA, respectively (Figure 1).

There was some variation between regions, with 'slows progress of disability' experiencing significant growth in the USA, positioning as a more frequent treatment driver than in the 5EU (5EU: 5.4\%; USA: 17.8\%). This highlights a potential shift in focus toward more long-term treatment goals in the therapeutic approach for US RRMS patients. Convenience-related factors, other than 'oral administration', were uncommon (e.g., 'frequency of administration': 5.7 and $2.1 \%$ in the 5EU and the USA, respectively; a factor which has remained of low importance in the USA between Q4 2015 and Q4 2017). Across both regions, efficacy factors were more frequently cited at later lines of treatment, compared with first line (1 L) (Figure 2). Although treatment decisions were largely efficacy-focused, where convenience factors such as 'oral administration' were cited as treatment drivers, this was more likely to occur earlier in the treatment pathway as both regions experience a decline in convenience-related drivers, in favor of efficacy, toward later lines of therapy.

Across all lines of therapy, treatment choices were most likely to be made jointly between physicians and patients in both the 5EU and the USA. While the choice of DMT at all lines remains predominantly the consequence of a joint decision, patient involvement decreases towards later lines of treatment. By third line $(3 \mathrm{~L}), 6.5$ and $12.4 \%$ of treatment decisions were the choice of the patient in the 5EU and the USA, respectively, in comparison to 9.0 and $17.5 \%$ at $1 \mathrm{~L}$ (Figure $3 \mathrm{~A}$ ). Physician-led treatment decisions were of a higher prevalence in the $5 \mathrm{EU}$ while the USA saw greater patient engagement at all lines of therapy, with engagement at $1 \mathrm{~L}$ outweighing the influence of a physician alone. A similar pattern was evident across all disease severities (mild, moderate and severe); the majority of treatment choices were jointly made. In comparison to the 5EU, USA treatment choices were less likely to be solely physician led (Figure 3B). However, for the most severe patients, physician influence is high (36.4 and 38.2\% in 5EU and USA, respectively) with minimal patient-only decision making in both regions (3.9 and 5.9\%).

\section{Discussion}

The results showed that treatment decisions were similar in the 5EU and the USA; both remained largely efficacyfocused across the 2015-2017 time period, although the convenience of an oral treatment was also an important factor in the market. RRMS patients in the 5EU and the USA were engaged in treatment decisions, particularly at the early stage of the treatment pathway, with the majority of treatment choices being made jointly by physicians and 


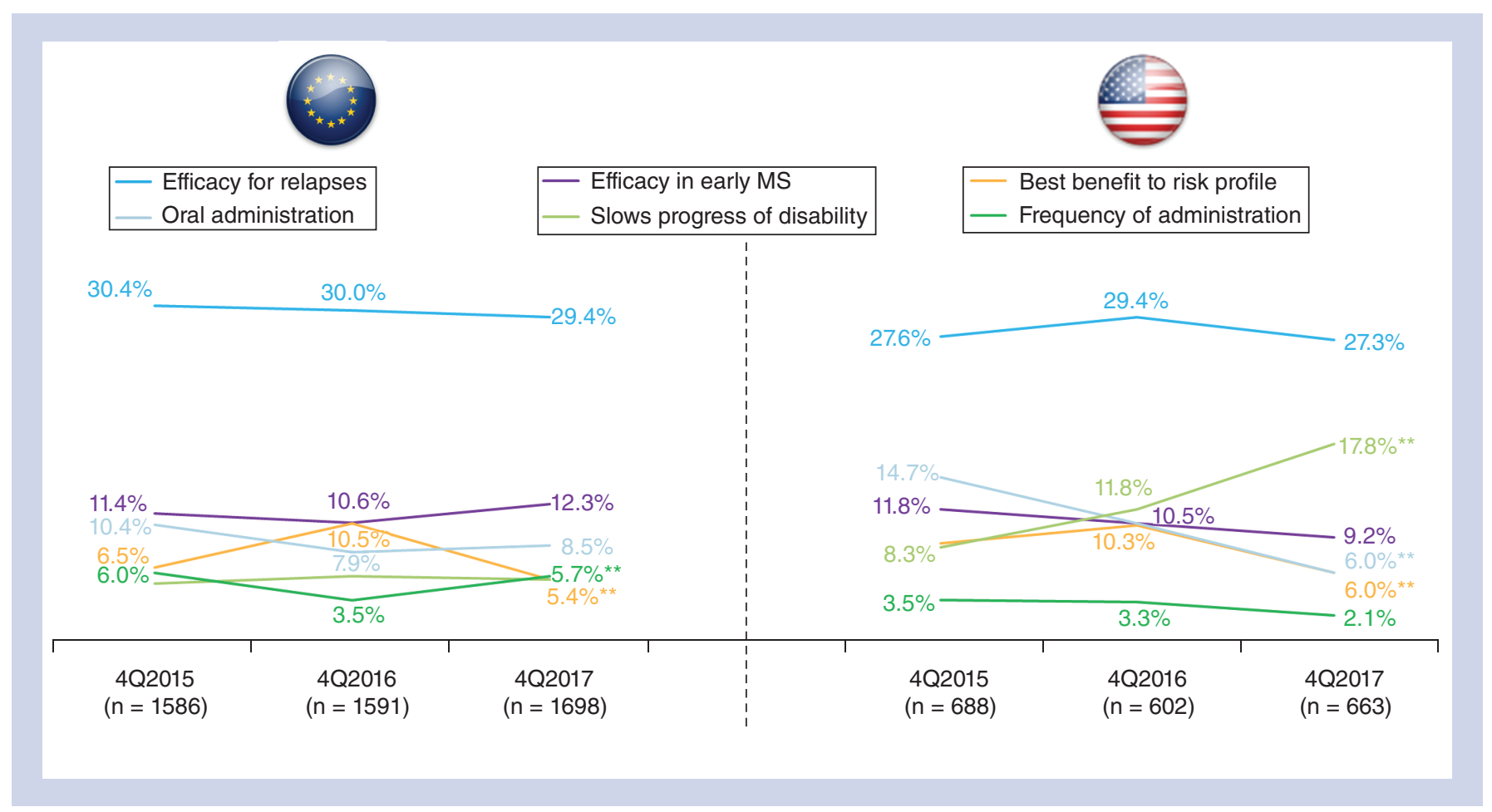

Figure 1. Primary reason for choice of current disease modifying therapy among relapsing-remitting multiple sclerosis patients between the 5EU and the USA over time.

**Sig. difference between 4Q2016 and 4Q2017 at $p<0.01$.

5EU: UK/Germany/France/Italy/Spain.

Data are (c) Ipsos 2018, all rights reserved.

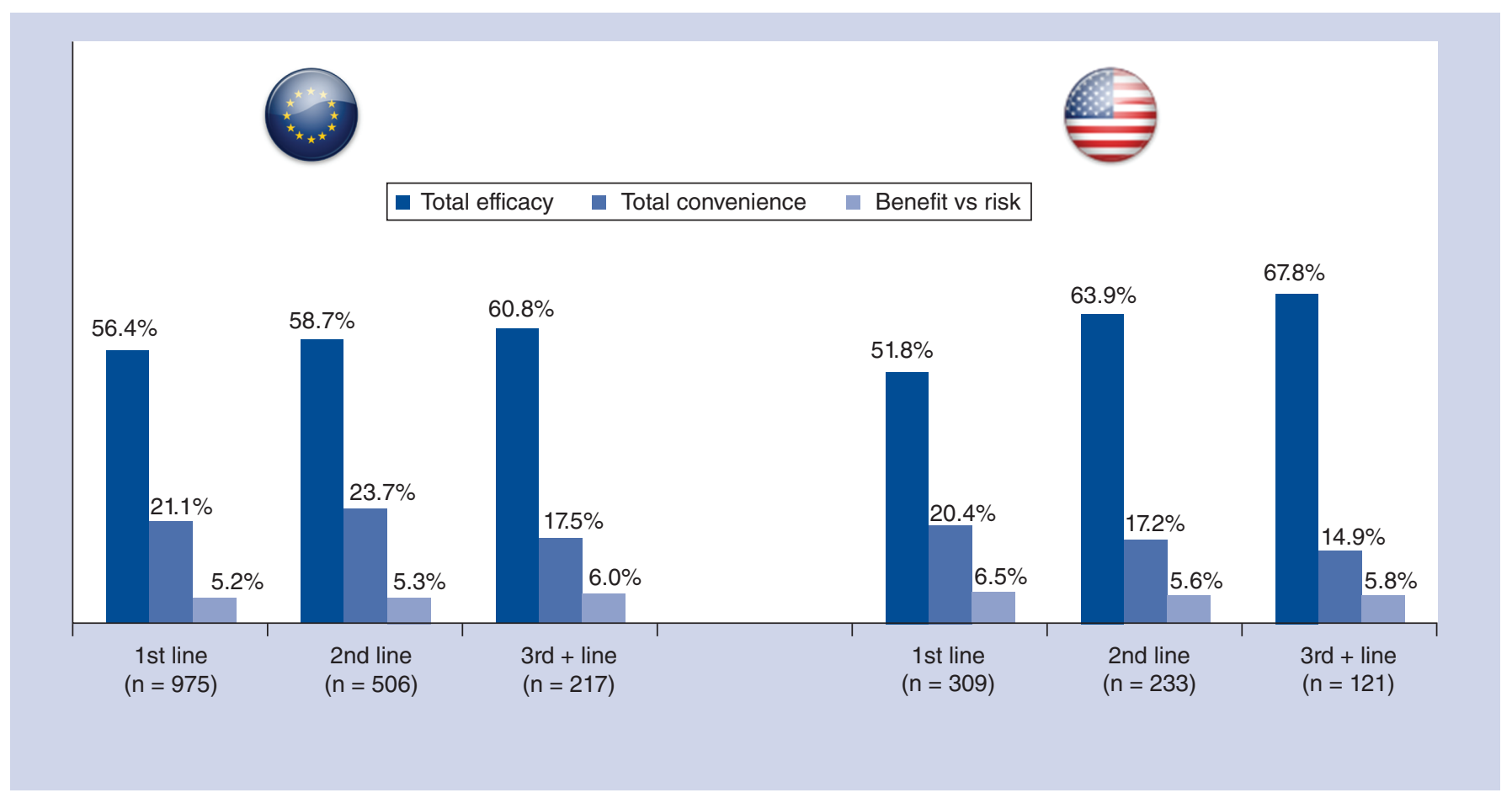

Figure 2. Primary reason for choice of current disease modifying therapy by lines in the 5EU and the USA; $4 Q 2017$.

5EU: UK/Germany/France/Italy/Spain.

Data are (C) Ipsos 2018, all rights reserved. 

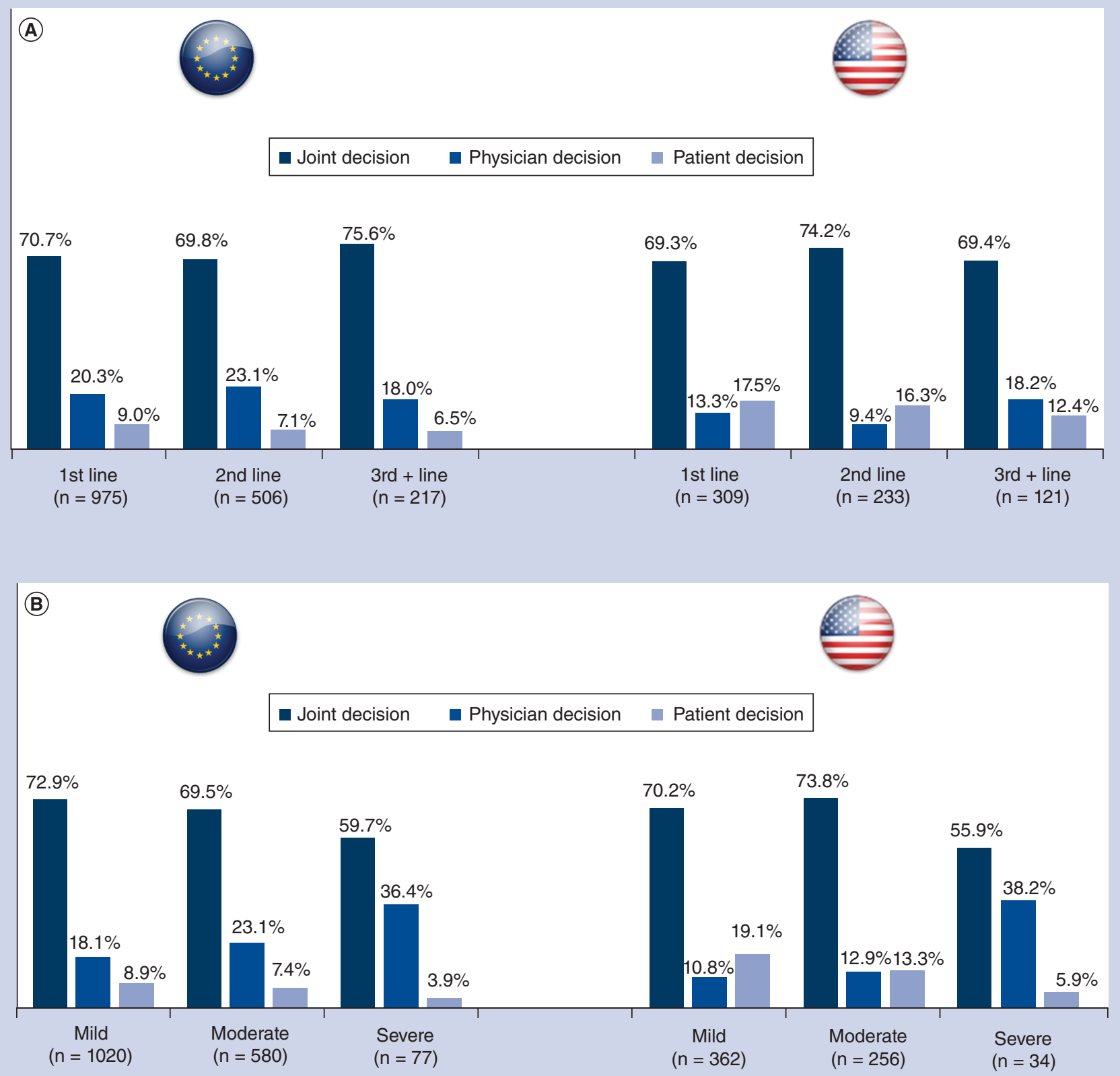

Figure 3. Physician and patient influence on treatment decisions in relapsing-remitting multiple sclerosis patient populations in the $5 E U$ and the USA. (A) Who drives treatment decisions by line of therapy in the 5EU and the USA. (B) Who drives treatment decisions by disease severity in the 5EU and the USA.

5EU: UK/Germany/France/Italy/Spain.

Data are (c) Ipsos 2018, all rights reserved.

patients. From what we can conclude for this study cohort, as patients progress through lines of therapy, treatment decisions are less likely to be patient-led, which is similar to patterns seen as patients' disease state becomes more severe. This decreasing patient involvement in line with disease progression may indicate a requirement for greater clinician intervention as a patient becomes faced with a reduced pool of treatment options. 
The benefits of patient involvement in treatment decisions are highlighted in several studies $[4,5]$. Patient engagement in treatment decision making, in conjunction with open dialogue around treatment options with physicians, is shown to have substantial influence on DMT satisfaction [4]. Additionally, with therapeutic options in MS being disease-modifying as opposed to curative, patient involvement in these critical interactions has shown to be essential for optimal adherence and persistence on therapy [5]. There is currently little evidence of RRMS patient engagement in treatment decisions as they progress through lines of therapy, hence this study is the first of its kind.

As the MS therapeutic market grows and newer treatment options become available, additional studies to supplement the evidence from this study cohort, in which efficacy remains paramount as a driver of choice, are vital. While 'efficacy for relapses' remains the dominant market driver, other influences such as 'benefit versus risk' continue to persist, as the growing adoption of riskier options does accompany something of a trade-off between the two. The requirement for a therapy with maximum efficacy and minimum risk is ever present [6].

The main limitation of this study is that the results are limited to the data collected and are therefore only representative of the physicians who completed the survey, their perceptions and their reported patients. There may be other uncollected factors which contribute to treatment decision making among RRMS patients in Europe and the USA. Of additional note; the study methodology utilises physician-reported patient data and therefore treatment drivers and patient engagement are based upon physician interpretation.

\section{Conclusion}

RRMS patients have a high level of engagement in their treatment decisions across both the EU5 and the USA, particularly early in the treatment pathway, with the majority of treatment choices made jointly by patients and physicians and driven by efficacy.

\section{Acknowledgments}

Data contained within this article are from the Ipsos Healthcare MS Therapy Monitor, used with permission. (C) Ipsos 2018, all rights reserved. H Brown, S Gabriele and J White were involved in drafting and reviewing the manuscript and supervising the data analysis.

Financial \& competing interests disclosure

The authors have no relevant affiliations or financial involvement with any organization or entity with a financial interest in or financial conflict with the subject matter or materials discussed in the manuscript. This includes employment, consultancies, honoraria, stock ownership or options, expert testimony, grants or patents received or pending, or royalties.

No writing assistance was utilized in the production of this manuscript.

Ethical conduct of research

The authors state that physicians provided written informed consent for the collection of data prior to study entry.

\section{Open access}

This work is licensed under the Attribution-NonCommercial-NoDerivatives 4.0 Unported License. To view a copy of this license, visit http://creativecommons.org/licenses/by-nc-nd/4.0/

\section{References}

Papers of special note have been highlighted as: $\bullet$ of interest

1. Lugaresi A, Di Loia M, Travaglini D, Pietrolongo E, Pucci E, Onofrj M. Risk-benefit considerations in the treatment of relapsing-remitting multiple sclerosis. Neuropsychiatr. Dis. Treat. 2013(9), 893-914 (2013).

2. Mehr SR, Zimmerman MP. Reviewing the unmet needs of patients with multiple sclerosis. Am. Health Drug Benefits 8(8), 426-431 (2015).

3. Colligan E, Metzler A, Tiryaki E. Share decision-making in multiple sclerosis. Mult. Scler. J. 23(2), 185-190 (2016).

4. Tintore M, Alexander M, Costello K et al. The state of multiple sclerosis: current insight into the patient/health care provider relationship, treatment challenges, and satisfaction. Patient Prefer. Adher. 2017(11), 33-45 (2016).

5. Lizán L, Comellas M, Paz S, Poveda JL, Meletiche DM, Polanco C. Treatment adherence and other patient-reported outcomes as cost determinants in multiple sclerosis: a review of the literature. Patient Prefer. Adher. 2014(8), 1653-1664 (2014). 
- The authors investigated costs relating to adherence of disease modifying therapies in patients with multiple sclerosis. It concludes that patient engagement in treatment decisions enhances clinical outcomes by benefiting treatment adherence on therapy.

6. Bruck W, Gold R, Lund B et al. Therapeutic decisions in multiple sclerosis: moving beyond efficacy. JAMA Neurol. 70(10), 1315-1324 (2013).

- The authors investigated the extent to which a disease modifying therapy's safety profile relates to their pharmacological characteristics. The study concludes that while efficacy remains dominant, the choice of new therapies necessitates consideration of additional drivers in the growing complexity of treatment decisions. 